Revue musicale OICRM

\title{
Performing Captivity, Performing Escape. Cabarets and Plays from the Terezín/Theresienstadt Ghetto, de Lisa Peschel
}

\section{Jean-Philippe Michaud}

Volume 3, numéro 2, 2016

Mémoire musicale et résistance. Autour du Verfügbar aux Enfers de Germaine Tillion

URI : https://id.erudit.org/iderudit/1060113ar

DOI : https://doi.org/10.7202/1060113ar

Aller au sommaire du numéro

Éditeur(s)

OICRM

ISSN

2368-7061 (numérique)

Découvrir la revue

Citer ce compte rendu

Michaud, J.-P. (2016). Compte rendu de [Performing Captivity, Performing Escape. Cabarets and Plays from the Terezín/Theresienstadt Ghetto, de Lisa Peschel]. Revue musicale OICRM, 3(2), 165-169.

https://doi.org/10.7202/1060113ar d'utilisation que vous pouvez consulter en ligne. 


\title{
Performing Captivity, Performing Escape. Cabarets and Plays from the Terezin/Theresienstadt Ghetto, de Lisa Peschel

\author{
Londres, Seagull Books, 2014, 446 pages
}

\author{
Jean-Philippe Michaud
}

Mots clés: arts et vie culturelle des camps ; cabarets ; rire et parodie ;

Terezín/Theresienstadt ; théâtre et résistance.

Keywords: arts and cultural life in concentration camps; cabarets; laughter and parody; Terezín/Theresienstadt; theatre and resistance.

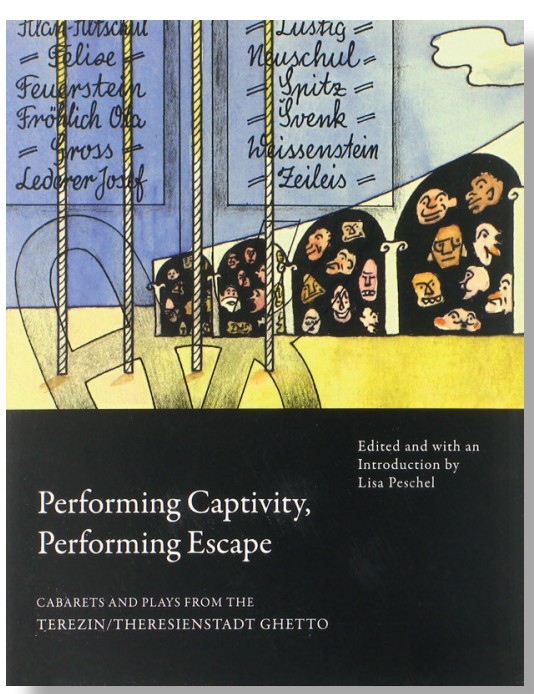

L'ouvrage présenté ici est un recueil de pièces et de textes scéniques rédigés lors de leur internement par des détenus du camp de concentration de Terezín/Theresienstadt. Lisa Peschel, chercheuse américaine spécialisée dans l'historiographie théâtrale et l'étude des matériaux artistiques produits durant l'Holocauste, avait déjà fait paraître à Prague en 2008 ce même dossier, dans la langue de rédaction des auteurs sélectionnés c'est-à-dire en allemand et en tchèque -, chaque texte étant systématiquement traduit dans l'autre langue. Divadelní texty $z$ terezinského ghetta/Theatertexte aus dem Ghetto Theresienstadt 1941-1945, ce travail d'édition bilingue issu de recherches dans différents fonds d'archives européens et israéliens, ainsi que d'entrevues avec des survivants du camp et des membres de la famille des artistes, insiste sur la dualité culturelle et le bilinguisme 
qui ont caractérisé la vie artistique de Terezín/Theresienstadt, dans les régions qui formeront la première République tchécoslovaque jusqu'à l'annexion allemande et durant la Seconde Guerre mondiale.

Déjà, une caractéristique majeure de ce camp est la concentration importante d'artistes professionnels et d'intellectuels tchécoslovaques, allemands et autrichiens qu'il a accueilli à partir de 1940. Situé entre Prague et la frontière actuelle avec l'Allemagne, le ghetto de Terezín/Theresienstadt est connu pour avoir été le lieu où la propagande nazie avait réussi, au plus fort de la guerre, à faire croire à la communauté internationale, lors d'une visite officielle de la Croix-Rouge, à l'existence d'une " colonie juive » (Jüdisches Siedlungsgebiet) offerte par le Führer. Cette formule euphémistique, utilisée pour taire les rumeurs sur l'extermination et l'horreur des camps, masque bien qu'en fait, Terezín/Theresienstadt a rempli tour à tour les fonctions de ghetto juif, de camp de concentration et de camp de transit vers les camps d'extermination à l'Est.

Par moments, le recueil aborde le ghetto sous l'angle des tensions culturelles et linguistiques ayant eu cours à l'époque en Europe centrale. Une certaine identité politique et culturelle juive s'est exprimée, dans les territoires slaves de Bohème et de Moravie au XIX ${ }^{e}$ siècle, par l'adoption de l'allemand comme langue d'usage et d'expression littéraire. Peschel expose assez amplement les causes des frictions identitaires entre communautés juives, mélange d'orgueil et de ressentiment, à la fin de l'Empire austro-hongrois et dans la République tchécoslovaque. Les modalités complexes des appartenances nationales et ethnoculturelles, après le tracé des nouvelles frontières, ont amené certains détenus à parodier sur scène, par exemple, la carence linguistique des Tchèques qui comprenaient mal l'allemand au camp, ou à critiquer à l'inverse le favoritisme et les avantages des germanophones à qui l'on confiait plus volontiers des postes de gestion interne du camp. Les pièces contenues dans l'ouvrage regorgent de renvois divers à ces dynamiques sociales et linguistiques, ainsi qu'à une foule de détails relevant de la vie quotidienne au camp, ce qui rend leur savante explicitation par l'éditrice d'autant plus importante.

Si le travail d'édition bilingue est écarté de la version unilingue anglaise de cet ouvrage - hormis certaines expressions et noms propres laissés dans leur langue originale -, sa riche introduction reconnaît dès les premières pages l'importance d'une méthodologie appropriée à l'analyse et à la mise en contexte de ce corpus duel de textes. Dans l'ensemble, on constate que l'effort de transposition et de traduction est réussi. Le système de notes atténue autant que possible l'ambiguïté des nombreux jeux de mots interlinguistiques et de trompe-oreilles humoristiques (obstacles majeurs à la traduction), tout en renvoyant souvent à une littérature et à une série d'entretiens contextuels encore largement inaccessibles au public anglophone (et francophone). Le glossaire bilingue, comprenant, en outre, quelques expressions yiddish, est cependant un expédient qui ne saurait compenser totalement l'absence des textes originaux.

Les documents publiés dans le recueil, au total cinq rédigés à l'origine en tchèque et sept en allemand, constituent des témoignages d'une valeur importante tant du point de vue de l'historiographie de la vie culturelle du camp que de celui, plus large, de la performance artistique comme mode de résistance en régime totalitaire. On est tout de suite frappé par la diversité des registres des pièces présentées ici. Plus 
encore, ce sont les multiples traces de l'organisation matérielle de ces efforts créatifs, à peine tolérés par l'administration du camp, qui saisissent le lecteur. Outre des dessins d'artistes, des dizaines d'illustrations accompagnent les écrits, dont celles des partitions et des scripts manuscrits, des couvertures des revues du ghetto, des affiches publicitaires des concerts indiquant les dates de représentations, des remerciements aux artistes, et même quelques tickets numérotés, rendant tangible cette situation irréelle où, prisonniers dans l'antichambre des camps de la mort, hommes, femmes et enfants mobilisent une vigoureuse puissance de liberté, de résistance et d'évasion.

En ce qui concerne la spécificité du camp, où durant une période les responsables nazis avaient même fait installer un kiosque pour orchestre dans le square principal, une remarque postérieure de Jana Šedová, l'une des comédiennes de la revue Prince Bettliegend (ou Le prince cloué au lit) reproduite dans le recueil, met en garde contre deux attitudes perverses et erronées que le regard historique se doit d'éviter. Premièrement, on ne devrait pas sous-estimer la souffrance des détenus sous prétexte que la production artistique supposerait des conditions de vie relativement tolérables ; deuxièmement, il faut également éviter de surfaire l'héroïsme des interprètes, la résistance dans les camps n'étant pas l'apanage d'une élite (p. 4). Ivan Klíma, autre survivant du camp, signe dans le prologue de l'ouvrage un témoignage de l'atmosphère étrange des concerts par lesquels les prisonniers pouvaient s'évader dans un autre monde (p. 38). Il rappelle aussi la présence récurrente du chant dans les baraques surpeuplées et de la musique accompagnant les tâches d'un quotidien des plus pénibles. Klíma évoque les raisons historiques, liées aux objectifs variables de propagande, pour lesquelles les autorités SS ont temporairement toléré - non sans sadisme - ces pratiques culturelles et permis qu'une telle concentration d'artistes, de peintres, de cinéastes, d'acteurs, de musiciens professionnels et de compositeurs puisse continuer à créer. Il souligne cependant le fait que toute mention directe des conditions réelles des prisonniers, et toute critique ouverte du traitement qu'ils recevaient au camp, enverraient aussitôt leur auteur à la mort.

La majorité des pièces musicales, théâtrales et d'opéra qui ont été représentées au camp, est tirée du répertoire " classique ", comme par exemple le célèbre Requiem de Verdi ${ }^{1}$, l'opéra-comique de Bedřich Smetana, La fiancée vendue, ou encore la riche production du compositeur Viktor Ullmann. L'ouvrage de Peschel donne cependant accès à une sélection de productions culturelles originales, d'un registre populaire aux accents plus subversifs. Cabarets, opérettes, drames historiques, spectacles musiqués et dansés, émissions de radio et satire juridique forment ensemble ce cortège scénique auquel nous convie Peschel.

Pour chacun des textes, l'appareil critique minutieux et efficace dans son développement dresse l'origine de chacun des manuscrits et de leurs variantes, avant d'établir la liste et la biographie des acteurs et des interprètes, des auteurs et des musiciens qui ont participé aux performances. Grâce à cet appareil critique se déploient, en parallèle,

1 L'ultime représentation menée par Rafael Schächter a reçu une attention spéciale dans le film documentaire de Doug Schultz, Defiant Requiem (2012). 
les traces des collaborations qu'ont entretenues certains des détenus, éclairant tant la chronologie des événements culturels que celle des réseaux d'amitiés qui s'y sont établis. Plutôt qu'un amas de textes divers et isolés qu'on aurait sortis des archives pour en faire un recueil, le tout contribue à faire de Performing Captivity, Performing Escape un ouvrage cohérent mettant habilement le doigt sur la valeur collective des productions scéniques à Terezín/Theresienstadt.

On y apprend que Hanuš Hachenburg, l'auteur de Hledáme strašidlo (Looking for a Specter), le spectacle de marionnettes à l'humour noir représenté depuis à Cape Town en 2001, n'avait que 13 ans à son arrivée au ghetto en 1942. Son jeune âge ne l'a pas empêché, pourtant, de s'établir comme poète dans la revue Vedem, éditée clandestinement par des enfants du camp, avant sa déportation à Auschwitz-Birkenau en décembre 1943. La pièce singulière se situe dans le royaume fictif d'Analphabète $1^{\mathrm{er}}$, personnage fantoche et ridicule représentant nul autre que le chef du Reich allemand vu par les yeux d'un enfant moqueur. Ce texte a récemment suscité une certaine attention en France et est paru en traduction sous le titre On a besoin d'un fantôme ${ }^{2}$.

L'une des pièces les plus remarquables du point de vue littéraire et compositionnel est le poème dramatique Orpheus (The Death of Orpheus) par lequel l'artiste de langue allemande, Georg Kafka, en reprenant le thème tragique d'Orphée, adresse une prière au héros à la mort duquel il ne pouvait que s'identifier. L'auteur, cousin éloigné du célèbre écrivain praguois homonyme, meurt dans l'un des transports du mois de mai 1944, mois durant lequel 7500 détenus ont été déportés à Auschwitz. Sa mère avait été sélectionnée pour le transport ; de façon volontaire et dans un ultime geste liant son destin à sa vision poétique, il résolut de l'accompagner aux portes de l'enfer.

Le spectacle de variétés Sméjte se s námi (Laugh with Us) contient l'un des passages touchant le plus près le désir illicite de nommer la réalité ténébreuse du camp. Dans ce scénario tchèque aux allures désinvoltes d'un cabaret comique autoréférentiel, Horpatzky, jouant au vieillard, est accosté parce qu'il porte encore une étoile - dans sa main. Un dialogue ironique sur un passé lointain et presque oublié s'établit :

Horpatzky : That was the time of the housing and food crisis, there wasn't much to eat and it was expensive, so we signed up and they transported us for free.

Porges : But where?

Horpatzky : Now what was that town called, named after some German poet, Schillero? No, something else. Who were the other German poets? [...]

Porges : Well, Goethe.

Horpatzky : Yes, correct, it was called ghetto.

Porges : Ghetto, ghetto? Wait, I read about that once in my school reader. But that was in the Middle Ages.

Horpatzky : Absolutely, and I lived through them as well. [...] these were modern Middle Ages with trains, tractors, telephones (p. 200-201).

2 Hanuš Hachenburg (2015), On a besoin d'un fantôme, édité et présenté par Claire Audhuy, Strasbourg, Rodéo d'âme. 
Vítězslav Horpatzky et Felix Prokeš (né Porges), duo comique issu du block A IV, étaient débarqués du même train en décembre 1941. Écrite et performée à l'été 1944, après plusieurs années de collaboration, la scène projetait le public dans une fiction d'après-guerre. Mais leur amitié rapidement établie annonçait déjà très tôt un penchant pour le cabaret et le commentaire historique, projetant sur l'actualité des prisonniers une mise à distance parodique. Leurs codétenus leur devaient aussi le Radio Show, spectacle nostalgique et bigarré également reproduit dans le recueil, représentant quant à lui les programmes radiophoniques praguois typiques d'avant-guerre. Leur attitude par rapport au camp anticipe et présuppose toujours la possibilité même du témoignage, c'est-à-dire la figuration d'une certaine continuité temporelle entre la vie « d'avant » et celle d'un possible « après » déportation, ainsi qu'une commensurabilité de l'expérience subjective du camp.

Dans cet esprit, les opérettes et cabarets performés à Terezín/Theresienstadt, parmi lesquels on retrouve édités aussi dans ce volume certaines pièces des cercles de Leo Straus (fils du compositeur viennois Oscar Straus) et de Hans Hofer, introduisent le plus souvent un souffle d'optimisme, d'humour et de divertissement bien essentiel. Il y a des raisons de croire qu'une pièce excessivement sombre et désespérée avait très peu de chance d'être mise en scène sur place. En 2006, Peschel apprit l'existence d'un tel inédit, d'une lucidité noire apparemment si prononcée qu'aucun interprète n'a eu le cœur de l'« infliger " aux détenus du camp. En exprimant dans leur pièce la certitude de ne retrouver après leur libération, à la fin de la guerre, qu'un monde irrémédiablement en ruines, les coauteurs du profond drame historique Dým domova (The Smoke of Home), à en croire Luděk Eliáš et ses compères (p. 147 s.), auraient rendu trop inconfortable la fine ligne qui sépare le jeu scénique, porteur d'espoir en ces pénibles circonstances, d'une entreprise dépourvue de sens dans un monde sans issue.

Le motif complexe de la résistance dans les camps, s'il occupe à lui seul un champ d'intérêt historiographique croissant, est cependant plus rarement envisagé depuis le vecteur des arts de la scène. Ce long travail d'archives et de recherche effectué en collaboration avec des survivants est en ce sens une contribution essentielle du théâtre de l'extrême, menant à une compréhension plus nuancée des rapports difficiles et ambigus des détenus entre eux et envers leurs bourreaux. Elle atteste une compréhension profonde de la situation particulière du camp " modèle » de Terezín/Theresienstadt, de sa vie culturelle, ainsi que de la rare détermination de ces femmes et de ces hommes à créer pour témoigner, et témoigner pour survivre. 